\title{
A MODEL OF THE BROAD-BAND CONTINUUM OF NGC 5548
}

\author{
P. MAGDZIARZ
}

Astronomical Observatory, Jagiellonian University

Orla 171, 30-244 Cracow, Poland

AND
O. BLAES

Department of Physics, University of California Santa Barbara, CA 93106, USA

\begin{abstract}
We discuss a model of the central source in Seyfert 1 galaxy NGC 5548. The model assumes a three phase disk structure consisting of a cold outer disk, a hot central disk constituting a Comptonizing $\mathrm{X} / \gamma$ source, and an intermediate unstable and complex phase emitting a soft excess component. The model qualitatively explains broad-band spectrum and variability behavior assuming that the soft excess contributes significantly to the continuum emission and drives variability by geometrical changes of the intermediate disk zone.
\end{abstract}

\section{Introduction}

Broad-band spectral analysis of NGC 5548 shows that a soft X-ray excess dominating below $1 \mathrm{keV}$ is related to the disk and may contribute significantly to the source energetics (Magdziarz et al. 1997). Since an optical/UV continuum requires the disk temperature of order a few $\mathrm{eV}$, the soft excess component needs an additional continuum emitting phase. The standard opticly thick accretion disk (Shakura \& Sunyaev 1973) breaks down at high accretion rate and, thus it puffs up producing the central, hot $(\sim 100$ $\mathrm{keV}$ ) region (e.g. Shapiro, Lightman \& Eardley 1976) which constitutes the Comptonizing $\mathrm{X} / \gamma$ source (e.g. Zdziarski et al. 1997). In a such model the third intermediate phase of the disk (with characteristic temperature $\sim 100$ $\mathrm{eV}$ ) naturally appears as an effect of an instability. 


\section{Physics of the central source}

Correlated variability of the spectral index, amount of reflection, and the total flux emitted in the $\mathrm{X} / \gamma$ continuum (Magdziarz et al. 1997) suggests that a number of seed photons controls the Compton cooling of the central $\mathrm{X} / \gamma$ source. This explains that the source is soft in a bright state and hard (i.e. photon starved) in a faint state. The opposite relation in the overall optical to soft excess continuum suggests that the main contributor of seed photons for Comptonization is the soft excess. Lack of ionized Compton reflection and lack of emission lines in EUV range requires complex structure of the soft excess. If the part of emitting matter is Thomson thin and dense it may emit dominant part of energy in a form of EUV pseudocontinuum (e.g. Kuncic, Celotti \& Rees 1997).

\section{Phenomenological model}

The model assumes that the observed variability is driven by geometrical changes of the unstable inner edge of the cold disk on thermal time scales (cf. Kaastra \& Barr 1989; Loska \& Czerny 1997). This edge couples to the hot plasma by energy reprocessing modulated with the solid angle of cold matter seen from the central hot disk. The instability of the intermediate zone between the cold and the hot phase puffs up the inner cold disk and leads to its fragmentation which may be responsible for the observed self-organized critically behavior of variability (e.g. Leighly 1997). The unstable zone has a complex structure of clouds or filaments which explains the observed continuum of cold Comptonization in the soft excess range (Magdziarz et al. 1997). The positive feedback between the number of seed photons emitted from the unstable inner edge of the cold disk and the energy radiated out from the hot central disk produces substantial nonlinearity in the variability and naturally explains the correlation of the total flux emitted in $\mathrm{X} / \gamma$ con inuum with the spectral index and amount of reflection. In such a physical picture, the soft excess dominantes energetics of the broad-band spectrum and drives variability of the central source.

\section{References}

Kaastra, J. S., \& Barr (1989) $A$ A 226, 59

Kuncic, Z., Celotti, A., \& Rees, M. J. (1997) MNRAS 284, 717

Leighly, K. M. (1997) this proceedings

Loska, Z., \& Czerny, B. (1997) MNRAS 284, 946

Magdziarz, P., et al. (1997) Proceedings of the 4-th Compton Symposium, in press

Shakura, N. I., \& Sunyaev, R. A. (1973) $A A 24337$

Shapiro, S. L., Lightman, A. P., \& Eardley, D. M. (1976) ApJ 204187

Zdziarski, A. A., et al. (1997) Proceedings of the 2nd INTEGRAL Workshop, ESA SP-382 